\title{
An efficient quantum algorithm for finding hidden parabolic subgroups in the general linear group
}

\author{
Thomas Decker * Gábor Ivanyos ${ }^{\dagger} \quad$ Raghav Kulkarni ${ }^{\ddagger}$ \\ Youming Qiao ${ }^{\S} \quad$ Miklos Santha ฯ
}

July 8, 2014

\begin{abstract}
In the theory of algebraic groups, parabolic subgroups form a crucial building block in the structural studies. In the case of general linear groups over a finite field $\mathbb{F}_{q}$, given a sequence of positive integers $n_{1}, \ldots, n_{k}$, where $n=n_{1}+\cdots+n_{k}$, a parabolic subgroup of parameter $\left(n_{1}, \ldots, n_{k}\right)$ in $\mathrm{GL}_{n}\left(\mathbb{F}_{q}\right)$ is a conjugate of the subgroup consisting of block lower triangular matrices where the $i$ th block is of size $n_{i}$. Our main result is a quantum algorithm of time polynomial in $\log q$ and $n$ for solving the hidden subgroup problem in $\mathrm{GL}_{n}\left(\mathbb{F}_{q}\right)$, when the hidden subgroup is promised to be a parabolic subgroup. Our algorithm works with no prior knowledge of the parameter of the hidden parabolic subgroup. Prior to this work, such an efficient quantum algorithm was only known for minimal parabolic subgroups
\end{abstract}

*EXASOL, Nuremberg, Germany (t.d3ck3r@gmail.com).

${ }^{\dagger}$ Institute for Computer Science and Control, Hungarian Academy of Sciences, Budapest, Hungary (Gabor.Ivanyos@sztaki.mta.hu).

${ }^{\ddagger}$ Centre for Quantum Technologies, National University of Singapore, Singapore (kulraghav@gmail.com).

${ }^{\S}$ Centre for Quantum Computation and Intelligent Systems, University of Technology, Sydney; and Centre for Quantum Technologies, National University of Singapore, Singapore (cqtqy@nus.edu.sg).

ILIAFA, Univ. Paris 7, CNRS, Paris, France; and Centre for Quantum Technologies, National University of Singapore, Singapore (miklos.santha@liafa.jussieu.fr). 
(Borel subgroups), for the case when $q$ is not much smaller than $n$ (G. Ivanyos: Quantum Inf. Comput., Vol. 12, pp. 661-669).

\section{Introduction}

Background. The hidden subgroup problem (HSP for short) is defined as follows. A function $f$ on a group $G$ is said to hide a subgroup $H \leq G$, if $f$ satisfies the following: $f(x)=f(y)$ if and only if $x$ and $y$ are in the same left coset of $H$ (that is, $x^{-1} y \in H$ ). When such an $f$ is given as a black box, the HSP asks to determine the hidden subgroup $H$. Note that the problem when the level sets of the hiding $f$ are demanded to be right cosets of $H$ - that is, $f(x)=f(y)$ if and only if $y x^{-1} \in H$ - is equivalent: composing $f$ with taking inverses maps a hiding function via right cosets to a hiding function via left cosets, and vice versa. When we explicitly want to refer to this variant of the problem, we speak about HSP via right cosets.

The complexity of a hidden subgroup algorithm is measured in terms of the number of bits representing the elements of the group $G$, which is usually $O(\log |G|)$. On classical computers, the problem has exponential query complexity even for abelian groups. In contrast, the quantum query complexity of HSP for any group is polynomial [10], and the HSP for abelian groups can be solved in polynomial time with a quantum computer [5, 21]. The latter algorithms are generalizations of Shor's result on order finding and computing discrete logarithms [24]. These algorithms can be further generalized to compute the structure of finite commutative black-box groups $[7]$.

To go beyond the abelian groups is well-motivated by its connection with the graph isomorphism problem. Despite considerable attention, the groups for which the HSP is tractable remain close to being abelian. For example, we know polynomial-time algorithms for the following cases: groups whose derived subgroups are of constant derived length and constant exponent [11], Heisenberg groups [2, 1] and more generally two-step nilpotent groups [19], "almost Hamiltonian" groups [12], and groups with a large abelian subgroup and reducible to the abelian case [16]. The limited success in going beyond the abelian case indicates that the nonabelian HSP may be hard, and [23] shows some evidence for this by providing a connection between the HSP in dihedral groups and some supposedly difficult lattice problem.

Instead of considering various ambient groups, another direction is to pose 
restrictions on the possible hidden subgroups. This can result in efficient algorithms, even over fairly nonabelian ambient groups. For example, if the hidden subgroup is assumed to be normal, then HSP can be solved in quantum polynomial time in groups for which there are efficient quantum Fourier transforms $[14,15]$, and even in a large class of groups, including solvable groups [18]. The methods of $[22,13]$ are able to find sufficiently large non-normal hidden subgroups in certain semidirect products efficiently.

Some restricted subgroups of the general linear groups were also considered in this context. The result by Denney, Moore and Russell in [8] is an efficient quantum algorithm that solves the HSP in the group of 2 by 2 invertible matrices (and related groups) where the hidden subgroup is promised to be a so-called Borel subgroup. In [17], Ivanyos considered finding Borel subgroups in general linear groups of higher degree, and presented an efficient algorithm when the size of the underlying field is not much smaller than the degree.

A well-known superclass of the family of Borel subgroups is the family of parabolic subgroups, whose definition is given below. In this work, we follow the line of research in $[8,17]$, and consider the problem of finding parabolic subgroups in general linear groups. Our main result will be a polynomialtime quantum algorithm for this case, without restrictions on field size.

Parabolic subgroups of the general linear group. Let $q$ be a power of a prime $p$. The field with $q$ elements is denoted by $\mathbb{F}_{q}$. The vector space $\mathbb{F}_{q}^{n}$ consists of column vectors of length $n$ over $\mathbb{F}_{q} . \mathrm{GL}_{n}\left(\mathbb{F}_{q}\right)$ stands for the general linear group of degree $n$ over $\mathbb{F}_{q}$. The elements of $\mathrm{GL}_{n}\left(\mathbb{F}_{q}\right)$ are the invertible $n \times n$ matrices with entries from $\mathbb{F}_{q}$. We also use $\operatorname{GL}(V)$ to denote the group of linear automorphisms of the $\mathbb{F}_{q}$-space $V$. With this notation, we have $\mathrm{GL}_{n}\left(\mathbb{F}_{q}\right) \cong \mathrm{GL}\left(\mathbb{F}_{q}^{n}\right)$ and throughout the paper we will identify these two groups. As a matrix is represented by an array of $n^{2}$ elements from $\mathbb{F}_{q}$, an algorithm is considered efficient if its complexity is polynomial in $n$ and $\log q$.

We now present the definition of parabolic subgroups (see [25]). For a positive integer $k$, and a sequence of positive integers $n_{1}, \ldots, n_{k}$ with $n_{1}+$ $\cdots+n_{k}=n$, the standard parabolic subgroup of $\mathrm{GL}_{n}\left(\mathbb{F}_{q}\right)$ with parameter $\left(n_{1}, \ldots, n_{k}\right)$ is the subgroup consisting of the invertible lower block triangular matrices of diagonal block sizes $n_{1}, \ldots, n_{k}$. Any conjugate of the standard parabolic subgroup is called a parabolic subgroup.

To see the geometric meaning of parabolic subgroups, we review the concept of flags of vector spaces. Let 0 also denote the zero vector space. For 
$\mathbb{F}_{q}^{n}$ and $k \geq 1$, a flag $F$ with the parameter $\left(n_{1}, \ldots, n_{k}\right)$ is a nested sequence of subspaces of $\mathbb{F}_{q}^{n}$, that is $\mathbb{F}_{q}^{n}=U_{0}>U_{1}>U_{2}>\cdots>U_{k-1}>U_{k}=0$, such that for $0 \leq i \leq k-1, \operatorname{dim}\left(U_{i}\right)=n_{i+1}+\cdots+n_{k}$. $k$ is called the length of $F$. For $g \in \mathrm{GL}_{n}\left(\mathbb{F}_{q}\right), g$ stabilizes the flag $F$ if for every $i \in[k], g\left(U_{i}\right)=U_{i}$. Then all group elements in $\mathrm{GL}_{n}\left(\mathbb{F}_{q}\right)$ stabilizing $F$ form a parabolic subgroup. On the other hand, any parabolic subgroup corresponds to some flag $F$, namely it consists of the elements in $\mathrm{GL}_{n}\left(\mathbb{F}_{q}\right)$ stabilizing $F$.

For example, the standard parablic subgroup $B$ in $\mathrm{GL}_{5}\left(\mathbb{F}_{q}\right)$ with parameter $(2,2,1)$ consists of invertible matrices of the form $\left(\begin{array}{lllll}* & * & 0 & 0 & 0 \\ * & * & 0 & 0 & 0 \\ * & * & * & * & 0 \\ * & * & * & * & 0 \\ * & * & * & * & *\end{array}\right)$. Let $\left\{e_{1}, \ldots, e_{5}\right\}$ be the standard basis of $\mathbb{F}_{q}^{5}$. The flag stabilized by $B$ is $\mathbb{F}_{q}^{5}>\left\langle e_{3}, e_{4}, e_{5}\right\rangle>\left\langle e_{5}\right\rangle>0$.

A parabolic subgroup is maximal if there are no parabolic subgroups properly containing it. It is minimal if it does not properly contain any parabolic subgroup. A parabolic subgroup $B$ in $\mathrm{GL}_{n}\left(\mathbb{F}_{q}\right)$ is maximal if and only if it is the stabilizer of a flag of length 2 , that is, it is the stabilizer of some nontrivial subspace. On the other hand, $B$ is minimal if it stabilizes a flag of length $n$. Borel subgroups in $\mathrm{GL}_{n}\left(\mathbb{F}_{q}\right)$ are just minimal parabolic subgroups. They are conjugates of the subgroup of invertible lower triangular matrices.

Our results. The main result of this paper is a polynomial-time quantum algorithm for finding parabolic subgroups in general linear groups.

Theorem 1. Any hidden parabolic subgroup in $\mathrm{GL}_{n}\left(\mathbb{F}_{q}\right)$ can be found in quantum polynomial time (i.e., in time poly $(\log q, n))$.

Note that this algorithm does not require one to know the parameter of the hidden parabolic subgroup in advance. Neither does it pose any restriction on the underlying field size, while the algorithm in [17] for finding Borel subgroups requires the field size to be large enough. The basic idea behind the algorithm is that in certain cases the superposition of the elements in a coset of the subgroup is close to a superposition of the elements of a linear space of matrices. The latter perspective allows the use of standard algorithms for abelian HSPs. Another crucial idea is to make use of the subgroup of common stabilizers of all the vectors on a random hyperplane, and examine its intersection with the hidden parabolic subgroup.

We state without proof the following result: consider certain subgroups of Borel subgroups, namely the full unipotent subgroups. They are conjugates of 
the subgroup of lower triangular matrices with 1's on the diagonal. Following a variant of the idea for Theorem 1, there exists an algorithm for finding full unipotent subgroups whose complexity is polynomial in $n$ and the field size. The structure of the paper. In Section 2 we collect certain preliminaries for the paper. In particular, in Section 2.2 we adapt the standard algorithm for abelian HSP to linear subspaces, which forms the basis of our algorithms. We then present an efficient quantum algorithm for finding maximal parabolic subgroups in Section 3. Section 4 describes a main technical tool, a generalization of the result of $[22,8]$ for finding complements in affine groups. In Section 5 we present the algorithm for finding parabolic subgroups, proving Theorem 1.

\section{Preliminary}

\subsection{Notations and facts}

Throughout the article, $q$ is a prime power. For $n \in \mathbb{N},[n]=\{1, \ldots, n\}$. $\mathcal{M}_{n}\left(\mathbb{F}_{q}\right)$ is the set of $n \times n$ matrices over $\mathbb{F}_{q}$. For a finite group $G$, we will be concerned with finding a subgroup $H$ in $G$, when it is promised that $H$ is from a fixed family of subgroups $\mathcal{H}$. We use $\operatorname{HSP}(G, \mathcal{H})$ to denote the HSP problem with this promise, and $\operatorname{rHSP}(G, \mathcal{H})$ to denote the $\mathrm{HSP}$ via right cosets of $H \in \mathcal{H}$. Let $V$ be a vector space. For a subspace $U \leq V$ and $G=\mathrm{GL}(V)$, let $G_{U}$ be the subgroup in $G$ consisting of elements that act as pointwise stabilizers on $U$. That is, $G_{U}=\{X \in \mathrm{GL}(V): \forall u \in U, X u=u\}$. Let $G_{\{U\}}$ be the subgroup in $G$ consisting of elements that act as setwise stabilizers on $U$. That is, $G_{\{U\}}=\{X \in \mathrm{GL}(V): X U=U\}$. Note that $\left\{G_{\{U\}}: 0<U<V\right\}$ is just the set of maximal parabolic subgroups.

Fact 1. For every prime power $q$, and for every positive integers $n \geq m$, the probability for a random $n \times m$ matrix $M$ over $\mathbb{F}_{q}$ to have rank $m$ is no less than what we have in the case of $q=2$, that is $\frac{1}{2} \cdot \frac{3}{4} \cdot \frac{7}{8} \cdots \approx 0.288788>1 / 4$.

\subsection{The quantum Fourier transform of linear spaces}

In this part we briefly discuss slight generalizations of the Fourier transform of linear spaces over $\mathbb{F}_{q}$ introduced in [17] and a version useful for certain

linear spaces of matrices. Let $V \cong \mathbb{F}_{q}^{m}$ be a linear space over the field $\mathbb{F}_{q}$ and assume that we are given a nonsingular symmetric bilinear function 
$\phi: V \times V \rightarrow \mathbb{F}_{q}$. By $\mathbb{C}^{V}$ we denote the Hilbert space of dimension $q^{m}$ having a designated orthonormal basis consisting of the vectors $|v\rangle$ indexed by the elements $v \in \mathbb{F}_{q}^{m}$.

Let $q=p^{r}$ where $p$ is a prime and let $\omega$ be the primitive $p$ th root $e^{\frac{2 \pi i}{p}}$ of unity. We define the quantum Fourier transform with respect to $\phi$ as the linear transformation $Q F T_{\phi}$ of $\mathbb{C}^{V}$ which maps

$$
|v\rangle \text { to } \frac{1}{\sqrt{|V|}} \sum_{u \in V} \omega^{\operatorname{Tr}(\phi(u, v))}|u\rangle,
$$

where $v \in V$ and $\operatorname{Tr}$ is the trace map from $\mathbb{F}_{q}$ to $\mathbb{F}_{p}$ defined as $\operatorname{Tr}(x)=$ $\sum_{i=0}^{r-1} x^{p^{i}}$. It turns out that $Q F T_{\phi}$ is a unitary map and, if the vectors from $V$ are represented by arrays of elements from $\mathbb{F}_{q}$ that are coordinates in terms of an orthonormal basis of $V$ with respect to $\phi$ (that is, $\phi$ is the standard inner product of $\mathbb{F}_{q}^{m}$ ) then $Q F T_{\phi}$ is just the $m$ th tensor power of the QFT defined in $[9]$ for $\mathbb{F}_{q}$. (This is the linear transformation of $\mathbb{C}^{\mathbb{F}_{q}}$ that maps $|x\rangle\left(x \in \mathbb{F}_{q}\right)$ to $\frac{1}{\sqrt{q}} \sum_{y \in \mathbb{F}_{q}} \omega^{\operatorname{Tr}(x y)}|y\rangle$.) Therefore, in this case, by Lemma 2.2 of [9], $Q F T_{\phi}$ has a polynomial time approximate implementation on a quantum computer. In the general case, where elements of $V$ are represented by coordinates in terms of a not necessarily orthonormal basis w.r.t. $\phi$, the map $Q F T_{\phi}$ can be efficiently implemented by composing the above transform with linear transformations of $\mathbb{C}^{V}$ corresponding to appropriate basis changes for $V$.

For a subset $A \subseteq V$ we adopt the standard notation $|A\rangle$ for the uniform superposition of the elements of $A$, that is $|A\rangle=\frac{1}{\sqrt{|A|}} \sum_{a \in A}|a\rangle$. Assume that we receive the uniform superposition $\left|v_{0}+W\right\rangle=\frac{1}{\sqrt{|W|}} \sum_{v \in W}\left|v_{0}+v\right\rangle$ over the a coset $v_{0}+W$ of the $\mathbb{F}_{q}$-linear subspace $W$ of $V$ and for some $v_{0} \in V$. Let $W^{\perp}$ stand for the subspace of $V$ consisting of the vectors $u$ from $\mathbb{F}_{q}^{m}$ such that $\phi(u, v)=0$ for every $v \in W$. By results from [17], if we measure the state after the Fourier transform, we obtain a uniformly random element of $W^{\perp}$. If instead of the uniform superposition over the coset $v_{0}+W$ we apply the QFT to the superposition $\left|v_{0}+W^{\prime}\right\rangle=\frac{1}{\sqrt{\left|W^{\prime}\right|}} \sum_{v \in W^{\prime}}\left|v_{0}+v\right\rangle$ over a subset $v_{0}+W^{\prime}$ for $\emptyset \neq W^{\prime} \subseteq W$, the resulting state is $\sum_{u \in V} c_{u}^{\prime}|u\rangle$, where

$$
c_{u}^{\prime}=\left\langle u\left|Q F T_{\phi}\right| v_{0}+W^{\prime}\right\rangle=\frac{\omega^{\operatorname{Tr} \phi\left(v_{0}, u\right)}}{\sqrt{\left|W^{\prime}\right||V|}} \sum_{v \in W^{\prime}} \omega^{\operatorname{Tr} \phi(v, u)} .
$$


For $u \in W^{\perp}$ we have

$$
\left|c_{u}^{\prime}\right|=\frac{\left|W^{\prime}\right|}{\sqrt{\left|W^{\prime}\right||V|}}=\frac{\sqrt{\left|W^{\prime}\right|}}{\sqrt{|W|}} \cdot \frac{1}{\sqrt{\left|W^{\perp}\right|}},
$$

whence, after measurement the chance of obtaining a particular $u \in W^{\perp}$ is

$\frac{\left|W^{\prime}\right|}{|W|}$ times as much as if we had in the case of the uniform distribution over $W^{\perp}$.

In this paper we consider subspaces and certain subsets of the linear space $\mathcal{M}_{n}\left(\mathbb{F}_{q}\right)$. If we take the inner product $\phi_{0}(A, B)=\operatorname{tr}\left(A B^{T}\right)$ the elementary matrices form an orthonormal basis. It follows that $Q F T_{\phi_{0}}$, being just the $n^{2}$ th tensor power of the QFT of $\mathbb{F}_{q}$, can be efficiently approximated. However, for the purposes of this paper it turns out to be more convenient using the inner product $\phi(A B)=\operatorname{tr}(A B)$. The map $Q F T_{\phi}$ is the composition of $Q F T_{\phi_{0}}$ with taking transpose (the latter is just a permutation of the matrix entries). The main advantage of considering $Q F T_{\phi}$ is that it is invariant in the following sense: we always obtain the same $Q F T_{\phi}$ even if we write matrices of linear transformations of the space $V=\mathbb{F}_{q}^{n}$ in terms of various bases. In particular, in our hidden subgroup algorithms we can think of our matrices in terms of a basis a priori unknown to us in which the hidden subgroup has a natural form, for example lower block triangular.

\subsection{A common procedure for HSP algorithms}

Suppose we want to find some hidden subgroup $H$ in $G=\mathrm{GL}_{n}\left(\mathbb{F}_{q}\right)$. Let $V=$ $\mathbb{F}_{q}^{n}$. We present the standard procedure that produce a uniform superposition over a coset of the hidden subgroup. This part will be common in (most of) the hidden subgroup algorithms presented in this paper. First we show how to produce the uniform superposition over $\mathrm{GL}(V)$. The uniform superposition $\frac{1}{q^{n^{2}}} \sum_{X \in \mathcal{M}_{n}\left(\mathbb{F}_{q}\right)}|X\rangle$ over $\mathcal{M}_{n}\left(\mathbb{F}_{q}\right)$ can be produced using the QFT for $\mathbb{F}_{q}^{n^{2}}$. Then, in an additional qubit we compute a Boolean variable according to whether or not the determinant of $X$ is zero. We measure this qubit, and abort if it indicates that the matrix $X$ has determinant zero. This procedure gives the uniform superposition over $\mathrm{GL}(V)$ with success probability more than $\frac{1}{4}$.

Next we assume that we have the uniform superposition $\frac{1}{\sqrt{|\mathrm{GL}(V)|}} \sum_{X}|X\rangle|0\rangle$, summing over $X \in \mathrm{GL}(V)$. Recall that $f$ is the function hiding the subgroup. We appended a new quantum register, initialized to zero, for holding the 
value of $f$. We compute $f(X)$ in this second register, measure and discard it. The result is $|A H\rangle=\frac{1}{\sqrt{|H|}} \sum_{X \in H}|A X\rangle$ for some unknown $A \in \mathrm{GL}(V)$. $A$ is actually uniformly random, but in this paper we will not make use of this fact.

\section{Maximal parabolic subgroups}

In this section, we settle the HSP when the hidden subgroup is a maximal parabolic subgroup, which will be used in the main algorithm in Section 5. It also helps to illustrate the idea of the idea of approximating a subgroup in the general linear group by a subspace in the linear space of matrices.

Recall that a parabolic subgroup $H$ is maximal if it stabilizes some subspace $0<U<\mathbb{F}_{q}^{n}$. We mentioned in Section 2.1 that they are just setwise stabilizers of subspaces. Determining $H$ is equivalent to finding $U$. Set $V=\mathbb{F}_{q}^{n}$.

Proposition 1. Let $G=\mathrm{GL}_{n}\left(\mathbb{F}_{q}\right)$, and $\mathcal{H}=\left\{G_{\{U\}}: 0<U<V\right\}$. $\operatorname{HSP}(G, \mathcal{H})$ can be solved in quantum polynomial time.

Proof. Let $H$ be the hidden maximal parabolic subgroup, stabilizing some $(n-d)$-dimensional subspace $U \leq \mathbb{F}^{n}$. Note that $d$ is unknown to us. Before describing the algorithm, we observe the following: checking correctness of a guess for $U$, and hence for $H$, can be done by applying the oracle to generators of the stabilizer of $U$, as there are no inclusions between maximal parabolic subgroups.

Now we present the algorithm. First produce a coset superposition $|A H\rangle$ for unknown $A \in \mathrm{GL}(V)$, as described in Section 2.3. Let $W=\{X \in$ $\left.\mathcal{M}_{n}\left(\mathbb{F}_{q}\right): X U \leq U\right\}$. In a basis whose last $n-d$ elements are from $U, W$ is the subspace of the matrices of the form $\left(\begin{array}{ll}B & \\ C & D\end{array}\right)$, where $B$ and $C$ are not necessarily invertible, and the empty space in the upper right corner means a $d \times(n-d)$ block of zeros. Noting that such a matrix is invertiable if and only if $B$ and $C$ are both invertible, we have $H \subset W$ and $\frac{|A H|}{|A W|}=\frac{|H|}{|W|}>\frac{1}{4 \times 4}$. Also, viewing in a basis in which $W$ is block triangular, $(A W)^{\perp} A$ consists of the matrices of the form $(*)$, where $*$ stands for an arbitrary $(n-d)$ times $d$ matrix. This implies that $(A W)^{\perp}=\left\{X \in \mathcal{M}_{n}\left(\mathbb{F}_{q}\right): X V \leq U\right.$ and $X U=$ 0) $A^{-1}$. 
If $d \geq n / 2$, we apply QFT to the left coset superposition $|A \mathcal{H}\rangle$ and perform a measurement, for any element $X$ in $(A W)^{\perp}$, the measurement will

produce $X$ with probability no less than $\frac{1}{16\left|(A W)^{\perp}\right|}$. It follows that $X A$ will be a particular matrix from $(A W)^{\perp} A$ with at least $\frac{1}{16\left|(A W)^{\perp}\right|}$. Then more than $\frac{1}{4}$ of the $(n-d) \times d$ matrices have rank $n-d$. It follows that with probability at least $\frac{1}{64}$, the matrix $X A$ will be a matrix from $(A W)^{\perp} A$ whose image is $U$. As $X V=X A V$, we can conclude that $X V=U$ with probability more than $\frac{1}{64}$.

For the case $d<n / 2$ we consider the HSP via right cosets of $H$, and let act matrices on row vectors from the right. Via the same procedure as above, it will reveal the dual subspace stabilized by $H$, which determines $H$ uniquely as well.

Finally, though $d$ is not known to us, depending on whether $d \geq n / 2$, one of these two procedures with produce $U$ correctly with high probability. So we perform the two procedures alternatively, and use the checking procedure to determine which produces the correct result. This concludes the algorithm.

\section{A tool: finding complements in small sta- bilizers}

In this section, we introduce and settle a new instance of the hidden subgroup problem. This will be an important technical tool for the main algorithm.

Consider the hidden subgroup problem in the following setting. The ambient group $G \leq \mathrm{GL}_{n}\left(\mathbb{F}_{q}\right)$ consists of the invertible matrices of the form $\left(\begin{array}{ll}b & \\ v & I\end{array}\right)$, where $b \in \mathbb{F}_{q}, v$ is a column vector from $\mathbb{F}_{q}^{n-1}$ and $I$ is the $(n-$ 1) $\times(n-1)$ identity matrix. The family of hidden subgroups $\mathcal{H}$ consists of all conjugates of $H_{0}$, where $H_{0}$ is the subgroup of diagonal matrices in $G: H_{0}=\left\{\left(\begin{array}{cc}b & \\ & I\end{array}\right): b \in \mathbb{F}_{q}^{*}\right\}$. Note that any conjugate of $H_{0}$ is $H_{v}=$ $\left\{\left(\begin{array}{cc}b & \\ (b-1) v & I\end{array}\right): b \in \mathbb{F}_{q}^{*}\right\}$, for some $v \in \mathbb{F}_{q}^{n-1}$. We will consider the HSP via right cosets in this setting.

The group $G$ has an abelian normal subgroup $N$ consisting of the matrices of the form $\left(\begin{array}{ll}1 & \\ v & I\end{array}\right)$ isomorphic to $\mathbb{F}_{q}^{n-1}$, and the subgroups $H_{v}$ are the 
semidirect complements of $N$. For $n=2, G$ is the affine group $\mathrm{AGL}_{1}\left(\mathbb{F}_{q}\right)$. The HSP in $\mathrm{AGL}_{1}\left(\mathbb{F}_{q}\right)$ is solved in quantum polynomial time in [22] over prime fields and in [8] in the general case using the non-commutative Fourier transform of the group $\mathrm{AGL}_{1}\left(\mathbb{F}_{q}\right)$. The algorithm served as the main technical ingredient in [8] for finding Borel subgroups in $\mathrm{GL}\left(\mathbb{F}_{q}^{2}\right)$. A generalization for certain similar semidirect product groups is given in [2]. To our knowledge, the first occurrence of the idea of comparing with a coset state in a related abelian group is in [2]. Here, due to the "nice" representation of the group elements, we can apply the same idea in a simpler way, while in [2] it was needed to be combined with a discrete logarithm algorithm which is not necessary here.

Proposition 2. Let $G$ and $\mathcal{H}$ be as above, and suppose $q=\Omega(n / \log n)$. Then $\operatorname{rHSP}(G, \mathcal{H})$ can be solved in quantum polynomial time.

Proof. Assume that the hidden subgroup is $H=H_{v}$ for some $v \in \mathbb{F}_{q}^{n-1}$. As right cosets of $H$ are being considered, we have superpositions over right cosets $H A$ for some unknown $A \in G$. The actual information of each matrix $X$ from $G$ is contained in $X-I$, a matrix from the $n$-dimensional space $L$ of matrices whose last $n-1$ columns are zero. We will work in $L$. Set

$\widetilde{W^{\prime}}=\{X-I: X \in H\}=\left\{\left(\begin{array}{c}b \\ b v\end{array}\right):-1 \neq b \in \mathbb{F}_{q}\right\}$ and $W=\left\{\left(\begin{array}{c}b \\ b v\end{array}\right): b \in \mathbb{F}_{q}\right\}$.

Then $W$ is a one-dimensional subspace of $L$. It turns out that $W=W A$ for every matrix $A \in G$ (that is why it is convenient to consider the HSP via right cosets). It follows that $\{(Y+I) A-I: Y \in W\}=\{Y A+(A-I)$ : $Y \in W\}=W+A-I$, whence the set $\{X A-I: X \in H\}=W^{\prime}+A-I$ for $W^{\prime}=\widetilde{W^{\prime}} A$.

Therefore, after an application of the QFT of $L$ to the state $|H A-I\rangle=$ $\left|W^{\prime}+A-I\right\rangle$ and a measurement, we obtain every specific element of $W^{\perp}$ with probability at least $\frac{q-1}{q} \frac{1}{\left|W^{\perp}\right|}$. More generally, if we do the procedure for a product of $n-1$ superpositions over right cosets of $H$ we obtain each specific $(n-1)$-tuple of vectors from $W^{\perp}$ with probability at least $\left(\frac{q-1}{q}\right)^{n-1} \frac{1}{\left|W^{\perp}\right|^{n-1}}$. Since the probability that $n-1$ random elements from a space of dimension $n-1$ over $\mathbb{F}_{q}$ span the space is at least $\frac{1}{4}$, therefore, the probability of getting a basis of $W^{\perp}$ is $\Omega\left(\left(\frac{q-1}{q}\right)^{n-1}\right)$. Using this basis, we obtain a guess for $W$ and $H$ as $H$ is the set of invertible matrices from $W+I$. A correct guess will be obtained expectedly with $O\left(\left(\frac{q}{q-1}\right)^{n-1}\right)$ repetitions. This is polynomial if $q$ is $\Omega(n / \log n)$. 
Finally we note that for constant $q$, or more generally for constant characteristic, [11] can be used to obtain a polynomial time algorithm. On the other hand, it is intriguing to study the case of "intermediate" values of $q$.

\section{The main algorithm}

\subsection{The structure of the algorithm}

In this subsection, we describe the structure of an algorithm for finding parabolic subgroups in general linear groups, proving Theorem 1 . Let $G=$ $\mathrm{GL}_{n}\left(\mathbb{F}_{q}\right), V=\mathbb{F}_{q}^{n}$, and the hidden parabolic subgroup $H$ be the stabilizer of the flag $V>U_{1}>U_{2}>\cdots>U_{k-1}>0$. Note that the parameter of the flag, including $k$, is unknown to us. The algorithm will output the hidden flag, from which a generating set of the parabolic subgroup can be constructed easily.

Let $T=U_{k-1}$ denote the smallest subspace in the flag. The algorithm relies on the following subroutines crucially. These two subroutines are described in Section 5.2 and Section 5.3, respectively.

Proposition 3. Let $G, H$ and $T$ be as above. There exists a quantum polynomial-time algorithm, that given access to an oracle hiding $H$ in $G$, produces three subspaces $W_{1}, W_{2}$ and $W_{3}$, s.t. one of $W_{i}$ is a nonzero subspace contained in $T$ with high probability.

Proposition 4. Let $G, H$ and $T$ be as above. There exists a classical polynomial-time algorithm, that given access to an oracle hiding $H$ in $G$, and some $0<W \leq V$, determines whether $W \leq T$, and in the case of $W \leq T$, whether $W=T$.

Given these two subroutines, the algorithm proceeds as follows. It starts with checking whether $k=1$, that is whether $H=G$. This can be done easily: produce a set of generators of $G$, and check whether the oracle returns the same on all of them. If $k=1$, return the trivial flag $V>0$.

Otherwise, it repeatedly calls the subroutine in Proposition 3 until that subroutine produces subspaces $W_{1}, W_{2}$ and $W_{3}$, such that for some $i \in[3]$, we have $0<W_{i} \leq T$. This can be verified by Proposition 4 . Let $W$ be this subspace. The second subroutine then also tells whether $W=T$.

After getting $0<W \leq T$, the algorithm fixes a subspace $W^{\prime}$ to be any direct complement of $W$ in $V$, and makes a recursive call to the HSP with 
a new ambient group $G^{\prime}$, and a new hidden subgroup $H^{\prime}$, as follows. $G^{\prime}$ is $\left\{X \in \mathrm{GL}(V): X W^{\prime} \leq W^{\prime}\right.$ and $\left.(X-I) W=0\right\}$, which is isomorphic to $\mathrm{GL}\left(W^{\prime}\right) \cong \mathrm{GL}(V / W)$. $H^{\prime}$ is the stabilizer of the flag $W^{\prime}>W^{\prime} \cap U_{1}>\cdots>$ $W^{\prime} \cap U_{k-1} \geq 0$. Note that the oracle restricted to $G^{\prime}$ realizes a hiding function for $H^{\prime}$.

The recursive call then returns a flag in $W^{\prime}$ as $W^{\prime}>U_{1}^{\prime}>U_{2}^{\prime}>\cdots>$ $U_{k^{\prime}}>0$. Let $U_{i}=\left\langle U_{i}^{\prime} \cup W\right\rangle, i \in\left[k^{\prime}\right]$. If $W=T$, then the algorithm outputs the flag $V>U_{1}>U_{2}>\cdots>U_{k^{\prime}}>W>0$. If $W<T$, return $V>U_{1}>U_{2}>\cdots>U_{k^{\prime}}>0$.

It is clear that at most $n$ recursive calls will be made, and the algorithm runs in polynomial time given that the two subroutines run in polynomial time too. We now prove Proposition 3 and 4 in the next two subsections.

\subsection{Guessing a part of the flag}

In this subsection we prove Proposition 3. Recall that $G=\mathrm{GL}_{n}\left(\mathbb{F}_{q}\right)$, the hidden subgroup $H$ stabilizing of the flag $V>U_{1}>\ldots>U_{k-1}>0$, and $T=U_{k-1}$. The algorithm of [8] for finding hidden Borel subgroups in 2 by 2 matrix groups was based on computing the intersection with the stabilizer of a nonzero vector. Here we follow an extension of the idea to arbitrary dimension $n$. We consider the common stabilizer of $n-1$ linearly independent vectors.

Pick a random subspace $U^{\prime} \leq V$ of dimension $n-1$. Recall that $G_{U^{\prime}}$ denotes the group of pointwise stabilizers of $U^{\prime}$. We also consider the group consisting of the unipotent elements of $G_{U^{\prime}}, N=\{X \in \mathrm{GL}(V):(X-I) V \leq$ $U^{\prime}$ and $\left.X \in G_{U^{\prime}}\right\}$. Note that $N$ is an abelian normal subgroup of $G_{U^{\prime}}$ of size $q^{n-1}$. Here we illustrate the form of $G_{U^{\prime}}$ and $N$ when $U^{\prime}$ is put in an appropriate basis:

$$
\left(\begin{array}{ccccc}
1 & & & & * \\
& 1 & & & * \\
& & 1 & & * \\
& & & 1 & * \\
& & & & *
\end{array}\right), \quad\left(\begin{array}{llllll}
1 & & & & * \\
& 1 & & & * \\
& & 1 & & * \\
& & & 1 & * \\
& & & & \\
& & G_{U^{\prime}} & & & \\
& & N & &
\end{array}\right) .
$$

We will describe three procedures, whose success on producing some $0<$ $W \leq T$ depend on $d:=\operatorname{dim}(T)$ and the field size $q$. Each of these procedures only works for a certain range of $d$ and $q$, but together they cover all possible cases. Thus, the algorithm needs to run each of these procedures, and return the three results from them. The general idea behind these procedures is 
to examine the intersection of the random hyperplane $U^{\prime}$ with $T$. As $d=$ $\operatorname{dim}(T)$, the probability that $U^{\prime}$ contains $T$ is $\frac{q^{n-d}-1}{q^{n}-1} \sim \frac{1}{q^{d}}$.

Assume first that $U^{\prime}$ does not contain $T$. We claim that in this case

$$
\begin{aligned}
\sum_{X \in H \cap G_{U^{\prime}}}(X-I) V & =T \\
\text { and } \sum_{X \in H \cap N}(X-I) V & =U^{\prime} \cap T .
\end{aligned}
$$

To see this, pick $v_{n} \in T \backslash U^{\prime}$, and let $v_{1}, \ldots, v_{n-1}$ be a basis for $U^{\prime}$ such that for every $0<j<k$, the system $v_{n-\operatorname{dim}\left(U_{j}\right)+1}, \ldots, v_{n-\operatorname{dim}\left(U_{j+1}\right)}$ is a basis for $U_{j}$. In the basis $v_{1}, \ldots, v_{n}$, the matrices of the elements of $N$ are the matrices with ones in the diagonal, arbitrary elements in the last column except the lowest one, and zero elsewhere. Among these the matrices of the elements of intersection with $H$ are those whose first $n-d$ entries in the last column are also zero:

$$
\left(\begin{array}{lllll}
* & & & & \\
* & * & & & \\
* & * & * & & \\
* & * & * & * & * \\
* & * & * & * & *
\end{array}\right), \quad\left(\begin{array}{llllll}
1 & & & & \\
& 1 & & & \\
& & 1 & & \\
& & & 1 & * \\
& & & & *
\end{array}\right),\left(\begin{array}{llllll}
1 & & & & \\
& 1 & & & \\
& & 1 & & \\
& & & 1 & * \\
& & & & 1
\end{array}\right) .
$$

Based on the above analysis, the three procedures are as follows.

- If $d>1$, then $H \cap N$ is nontrivial. As $N$ is abelian, we can efficiently compute $H \cap N$ by the abelian hidden subgroup algorithm. Thus by Equation 3 , we can use it to compute $W_{1}$ as a guess for a nontrivial subspace of $T$.

- If $d=1$ and $q \geq n$, we can compute $H \cap G_{U^{\prime}}$ in $G_{U^{\prime}}$ by the algorithm in Proposition 2, and use it to compute $W_{2}$ as a guess for $T$ by Equation 2.

- If $d=1$ and $q<n$, with probability at least $\frac{1}{q}-\frac{1}{q^{2}}=\Omega\left(\frac{1}{q}\right)=\Omega\left(\frac{1}{n}\right)$, we have that $U^{\prime} \geq T$ but $U^{\prime}$ does not contain $U_{k-2}$. Then we have

$$
\sum_{X \in H \cap N}(X-I) V=U^{\prime} \cap U_{k-2} .
$$

To see this, pick $v_{n} \in U_{k-1} \backslash\{0\}, v_{n-1} \in U_{k-2} \backslash U^{\prime}$, and $v_{1}, \ldots, v_{n-2}$ s.t. $v_{1}, \ldots, v_{n-2}, v_{n}$ is a basis for $U^{\prime}$ and for every $0<j<k$, the system $v_{n-\operatorname{dim}\left(U_{j}\right)+1}, \ldots, v_{n-\operatorname{dim}\left(U_{j+1}\right)}$ is a basis for $U_{j}$. In this basis the matrices for the elements of $N \cap H$ are those whose entries are zero except the 
ones in the diagonal and except the other lowest $\operatorname{dim} U_{k-2}$ entries in the next to last column:

$$
\left(\begin{array}{rrrrrr}
* & & & & \\
* & * & & & \\
* & * & * & * & \\
* & * & * & * & \\
* & * & * & * & *
\end{array}\right),\left(\begin{array}{rrrrr}
1 & & & * & \\
& 1 & & * & \\
& & 1 & * & \\
& & & 1 & \\
& & & * & 1
\end{array}\right),\left(\begin{array}{llllll}
1 & & & & \\
& 1 & & & \\
& & 1 & * & \\
& & & 1 & \\
& & & * & 1
\end{array}\right) .
$$

Again, we can find $H \cap N$ by the abelian hidden subgroup algorithm and use Equation 4 to compute $V^{\prime}=U^{\prime} \cap U_{k-2}$. If $\operatorname{dim} V^{\prime}=1$ then return $W_{3}=V^{\prime}$ as the guess for $T$. Otherwise we take a direct complement $V^{\prime \prime}$ of $V^{\prime}$ and restrict the HSP to the subgroup of the tranformations $X$ such that $(X-I) V^{\prime \prime}=0$ and $X V^{\prime} \leq V^{\prime}$ (which is isomorphic to $\left.\mathrm{GL}\left(V^{\prime}\right)\right)$ and apply the method in Proposition 1 to compute a subspace $W_{3}$ as the guess for $T$.

\subsection{Checking and recursion}

In this subsection we prove Proposition 4. Recall that the goal is to determine whether some subspace $0<W \leq V$ is contained in $T=U_{k-1}$, the last member of the flag $V>U_{1}>\cdots>U_{k-1}>0$ stabilized by the hidden parabolic subgroup $H$. If $W \leq V$, we'd like to know whether $W=T$. This can be achieved with the help of the following lemma, whose proof is omitted here.

Lemma 2. Let $H$ be the stabilizer in $\operatorname{GL}(V)$ of the flag $V>U_{1}>U_{2}>$ $\ldots>U_{k-1}>0$, and let $0<W<V$. Let $W^{\prime}$ be any direct complement of $W$ in $V$. Then $U_{k-1} \geq W$ if and only if $H \geq\{X \in \mathrm{GL}(V):(X-I) V \leq W\}$. Furthermore, if $U_{k-1} \geq W$ then $U_{k-1}=W$ if and only if

$$
H \cap\left\{X \in \mathrm{GL}(V):(X-I) V \leq W^{\prime} \text { and }(X-I) W^{\prime}=0\right\}=\{I\} .
$$

It is clear that this allows us to determine whether $U_{k-1} \geq W$ : form a generating set of $\{X \in \mathrm{GL}(V):(X-I) V \leq W\}$, and query the oracle to see whether all element in the generating set evaluate the same. Similarly if $U_{k-1} \geq W$, we can test whether $U_{k-1}=W$.

Acknowledgements. The research is partially funded by the Singapore Ministry of Education and the National Research Foundation, also through the Tier 3 Grant "Random numbers from quantum processes," MOE2012-T3-1009. Research partially supported by the European Commission IST STREP 
project Quantum Algorithms (QALGO) 600700, by the French ANR Blanc program under contract ANR-12-BS02-005 (RDAM project), and by the Hungarian Scientific Research Fund (OTKA), Grant NK105645.

\section{References}

[1] D. Bacon (2008), How a Clebsch-Gordan transform helps to solve the Heisenberg hidden subgroup problem, Quantum Inf. Comput., Vol. 8, pp. 438-467.

[2] D. Bacon, A. Childs, and W. van Dam (2005), From optimal measurement to efficient quantum algorithms for the hidden subgroup problem over semidirect product groups, In Proc. 46th IEEE FOCS, pp. 469-478.

[3] E. R. Berlekamp (1968), Algebraic coding theory, McGraw-Hill, New York.

[4] E. R. Berlekamp (1970), Factoring polynomials over large finite fields, Math. Comput., Vol. 24, pp. 713-735.

[5] D. Boneh and R. Lipton (1995), Quantum cryptanalysis of hidden linear functions, In: Proc. Crypto'95, pp. 427-437.

[6] D. G. Cantor, H. Zassenhaus (1981), A New Algorithm for Factoring Polynomials Over Finite Field, Math. Comput. 36, pp. 587-592.

[7] K. Cheung and M. Mosca (2001), Decomposing finite abelian groups, Quantum Inf. Comput., Vol. 1, pp. 26-32.

[8] A. Denney, C. Moore, and A. Russell (2010), Finding conjugate stabilizer subgroups in PSL(2; $)$ and related problems, Quantum Inf. Comput., Vol. 10, pp. 282-291.

[9] W. van Dam, S. Hallgren, and L. Ip (2006), Quantum algorithms for some hidden shift problems, SIAM J. Comput., Vol 36, pp 763-778.

[10] M. Ettinger, P. Hoyer, and E. Knill (2004), The quantum query complexity of the hidden subgroup problem is polynomial, Inform. Proc. Lett., 91, pp. 43-48. 
[11] K. Friedl, G. Ivanyos, F. Magniez, M. Santha, and P. Sen (2003), Hidden translation and orbit coset in quantum computing, In: Proc. 35th STOC, pp. 1-9.

[12] D. Gavinsky (2004), Quantum solution to the hidden subgroup problem for poly-near-Hamiltonian groups, Quantum Inf. Comput. Vol. 4, pp. $229-235$.

[13] D. N. Goncalves, R. Portugal and C. M. M. Cosme (2009), Solutions to the hidden subgroup problem on some metacyclic groups, In: Proc. TQC2009, Lect. Notes Comput. Sci., Vol. 5906, Springer-Verlag (Berlin), pp. 1-9.

[14] M. Grigni, L. Schulman, M. Vazirani, and U. Vazirani (2001), Quantum mechanical algorithms for the nonabelian Hidden Subgroup Problem, In Proc. 33rd ACM STOC, pp. 68-74.

[15] S. Hallgren, A. Russell, and A. Ta-Shma (2003), Normal subgroup reconstruction and quantum computation using group representations, SIAM J. Comp., 32, pp. 916-934.

[16] Y. Inui and F. Le Gall (2007), Efficient quantum algorithms for the hidden subgroup problem over semi-direct product groups, Quantum Inf. Comput., Vol. 7, pp. 559-570.

[17] G. Ivanyos (2012), Finding hidden Borel subgroups of the general linear group, Quantum Inf. Comput., Vol. 12, pp. 0661-0669.

[18] G. Ivanyos, F. Magniez, and M. Santha (2003), Efficient quantum algorithms for some instances of the non-Abelian hidden subgroup problem, Int. J. Found. Comp. Sci., Vol. 15, pp. 723-739.

[19] G. Ivanyos, L. Sanselme, and M Santha (2012), An efficient quantum algorithm for the hidden subgroup problem in nil-2 groups, Algorithmica 63(1-2): pp. 91-116.

[20] R. Jozsa (2001), Quantum factoring, discrete logarithms, and the hidden subgroup problem, Computing in Science and Engineering, Vol. 3, pp. 34-43. 
[21] A. Yu. Kitaev (1995), Quantum measurements and the Abelian Stabilizer Problem, Technical report arXiv:quant-ph/9511026.

[22] C. Moore, D. Rockmore, A. Russell, and L. Schulman (2004), The power of basis selection in Fourier sampling: Hidden subgroup problems in affine groups, In Proc. 15th ACM-SIAM SODA, pp. 1106-1115.

[23] O. Regev (2004), Quantum computation and lattice problems, SIAM J. Comput. 33, pp. 738-760.

[24] P. Shor (1997), Algorithms for quantum computation: Discrete logarithm and factoring, SIAM J. Comput., 26, pp 1484-1509.

[25] T. A. Springer (1998), Linear Algebraic groups, Progress in mathematics, Vol. 9, 2nd ed., Birkhäuser (Boston).

[26] J. Watrous (2001), Quantum algorithms for solvable groups, In Proc. 33rd ACM STOC, pp. 60-67. 